\title{
Localized vs. synchronized exports across a highly renewable pan-European transmission network
}

Rolando A. Rodriguez ${ }^{1 \dagger}$, Magnus Dahl ${ }^{2+}$, Sarah Becker ${ }^{3+}$ and Martin Greiner ${ }^{1,4^{*}+}$

\begin{abstract}
Background: A future, highly renewable electricity system will be largely based on fluctuating renewables. The integration of wind and solar photovoltaics presents a major challenge. Transmission can be used to lower the need for complementary generation, which we term backup in this article.

Methods: Generation data based on historical weather data, combined with real load data, determine hourly mismatch timeseries for all European countries, connected by physical power flows. Two localized export schemes determining the power flows are discussed, which export only renewable excess power, but no backup power, and are compared to a synchronized export scheme, which exports renewable excess power and also backup power.

Results: Compared to no or very limited power transmission, unconstrained power flows across a highly renewable pan-European electricity network significantly reduce the overall amount of required annual backup energy, but not necessarily the required backup capacities.

Conclusions: The reduction of the backup capacities turns out to be sensitive to the choice of export scheme. Results suggest that the synchronized export of local backup power to other countries is important to significantly save on installed backup capacities.
\end{abstract}

Keywords: Renewable energies, Energy system, Power transmission, Pan-European transmission grid

\section{Background}

Today's macro energy systems, mainly based on conventional fossil and nuclear resources, will in the future increasingly rely on renewable resources. At the moment, it is unclear what will be the best transitional pathway between the current and the future energy system. In this respect, it makes sense to think backwards, which means in a first step to get a good understanding of fully renewable energy systems [1-19] and then in a second step bridge to today's energy system [20,21]. Since wind and solar power generation are expected to be dominant, the fluctuating spatio-temporal weather patterns will determine the design of highly renewable energy systems.

\footnotetext{
*Correspondence: greiner@eng.au.dk

${ }^{\dagger}$ Equal contributors

1 Department of Mathematics, Aarhus University, Ny Munkegade 118, 8000 Aarhus C, Denmark

${ }^{4}$ Department of Engineering, Aarhus University, Inge Lehmanns Gade 10, 8000 Aarhus C, Denmark

Full list of author information is available at the end of the article
}

Based on state-of-the-art high-resolution meteorological data [22], spatio-temporal modeling, and the physics of complex networks [23, 24], some fundamental properties of a highly renewable pan-European power system have already been derived. Amongst such characteristics are the optimal mix of wind and solar power generation $[11,12]$, the synergies between storage and balancing [13], the extension of the transmission network $[14,20]$ as well as the ramp down of conventional power generation [21] during the transitional phase. These results indicate that the pathways into future energy systems will be driven by an optimal systemic combination of technologies that reduce the system's reliance on backup power and energy.

The benefit of transmission in highly renewable energy networks has been discussed and explored in [14, 20]. A simplified model of the electricity system has been considered, consisting of intermittent wind and solar PV production and a perfectly flexible dispatchable backup system. In this context, the backup energy, which is the

\section{黑 Springer}

(C) 2015 Rodriguez et al. This is an Open Access article distributed under the terms of the Creative Commons Attribution License (http://creativecommons.org/licenses/by/4.0), which permits unrestricted use, distribution, and reproduction in any medium, provided the original work is properly credited. 
integral over the usage of backup units in order to balance temporal deficits of the renewable power generation, turns out to be a strongly convex function of the overall transmission line capacities. For a fully renewable panEuropean power system with zero interconnector capacities, the required backup energy decreases from $24 \%$ of the annual electricity consumption down to $15 \%$ with sufficiently large transmission capacities which permit unconstrained power exchange. Most of this reduction is already achieved with transmission capacities three to four times larger than today's interconnector strengths. Although backup energy quickly decreases with increasing transmission capacities, it has been observed that the backup capacities are not similarly reduced [14].

The objective of this paper is to generalize the interplay between backup and transmission used in [14], and to discuss its potential for a combined reduction of backup energy and capacity in a highly renewable energy network. Figure 1 presents a toy model to clarify this objective. The first node in the triangular network (a) generates more renewable power than what it consumes; it has two units of renewable excess power. The other two nodes do not generate enough renewable power compared to what they consume, and have a deficit of three and two units. In (b), which is the export scheme used in $[14,20]$, the source node exports one excess unit to each of the two sink nodes, which then still have to balance two and one units. In (c), the source node also exports its two excess units, but now in such a way that after their imports, the two sink nodes have to balance the same amount of 1.5 units. In (d), the source node exports three units, which is more than

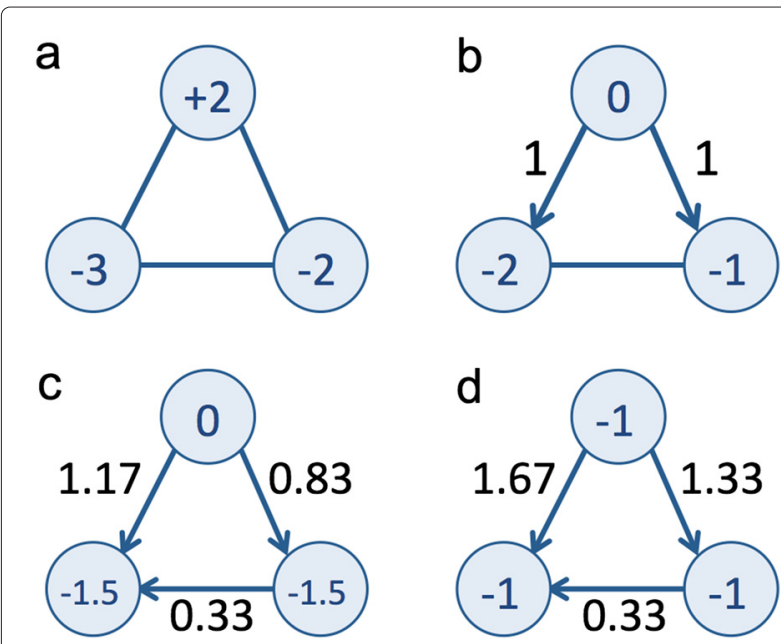

Fig. 1 Four export schemes. The network with one source and two sinks can be balanced via different export schemes: a zero exports, $\mathbf{b}$ maximum export of renewable excess power with minimum overall power flow, c maximum export of renewable excess power with maximum reduction of local backup, $\mathbf{d}$ combined export of renewable excess and backup power with maximum reduction of local backup its own excess power. Two units go to the second node and one unit to the third node. All three nodes are then left to balance one unit.

Behind schemes $(b-d)$ is Thomson's principle of minimal squared flow. After the maximum export of the source node's excess power has been chosen, the resulting power flows through the network are determined by squared flow minimization, in accordance with the physical power flow on the network. Since the role of the three nodes might swap due to the fluctuating weather patterns, (b) leads to an average network-wide backup energy of three units, a backup capacity of two units per node and a transmission capacity of one unit per link. In (c), after the maximum export of the source node's excess power, the resulting flow leads to an equal usage of backup capacity for the sink nodes. The scheme (c) also leads to a networkwide backup energy of three units, but when compared to (b), the backup capacity per node is only 1.5 units, whereas the transmission capacity per link has increased to 1.17 units. The shared backup principle of $(\mathrm{d})$ requires all nodes to balance the same amount after the imports and exports have taken place. It also keeps the networkwide backup energy to three units. The backup capacity at each node is reduced to one unit, and the transmission capacities of the links are increased to 1.67 units.

The three export schemes illustrated in Fig. $1 \mathrm{~b}-\mathrm{d}$ will be formalized in the "Methods" section. The "Results and discussion" section will use a simplified pan-European transmission network (see Fig. 2) to quantify the differences between the three export schemes with respect to backup energy, backup capacity, and transmission capacities. A conclusion will be given in the last section.

\section{Methods}

\section{Renewable power generation}

We define a country $n$ to have a fully renewable power system once the average power generation from renewable sources $\left\langle G_{n}^{R E S}\right\rangle=\left\langle L_{n}\right\rangle$ matches the average load. The renewable power generation

$$
G_{n}^{\mathrm{RES}}(t)=G_{n}^{W}(t)+G_{n}^{S}(t)
$$

is assumed to consist of wind $\left(G_{n}^{W}\right)$ and solar photovoltaics $\left(G_{n}^{S}\right)$. While large hydroelectric already offers significant amounts of renewable energy to the system, most growth is expected to take place in wind and solar. Due to the relative control possible in hydroelectric generation, we consider it as a part of dispatchable backup. Other renewable sources are either neglected (like run-ofriver hydro) or considered as part of the backup system (like biomass). The ratio

$$
\alpha_{n}=\left\langle G_{n}^{W}\right\rangle /\left\langle L_{n}\right\rangle=1-\left\langle G_{n}^{S}\right\rangle /\left\langle L_{n}\right\rangle
$$




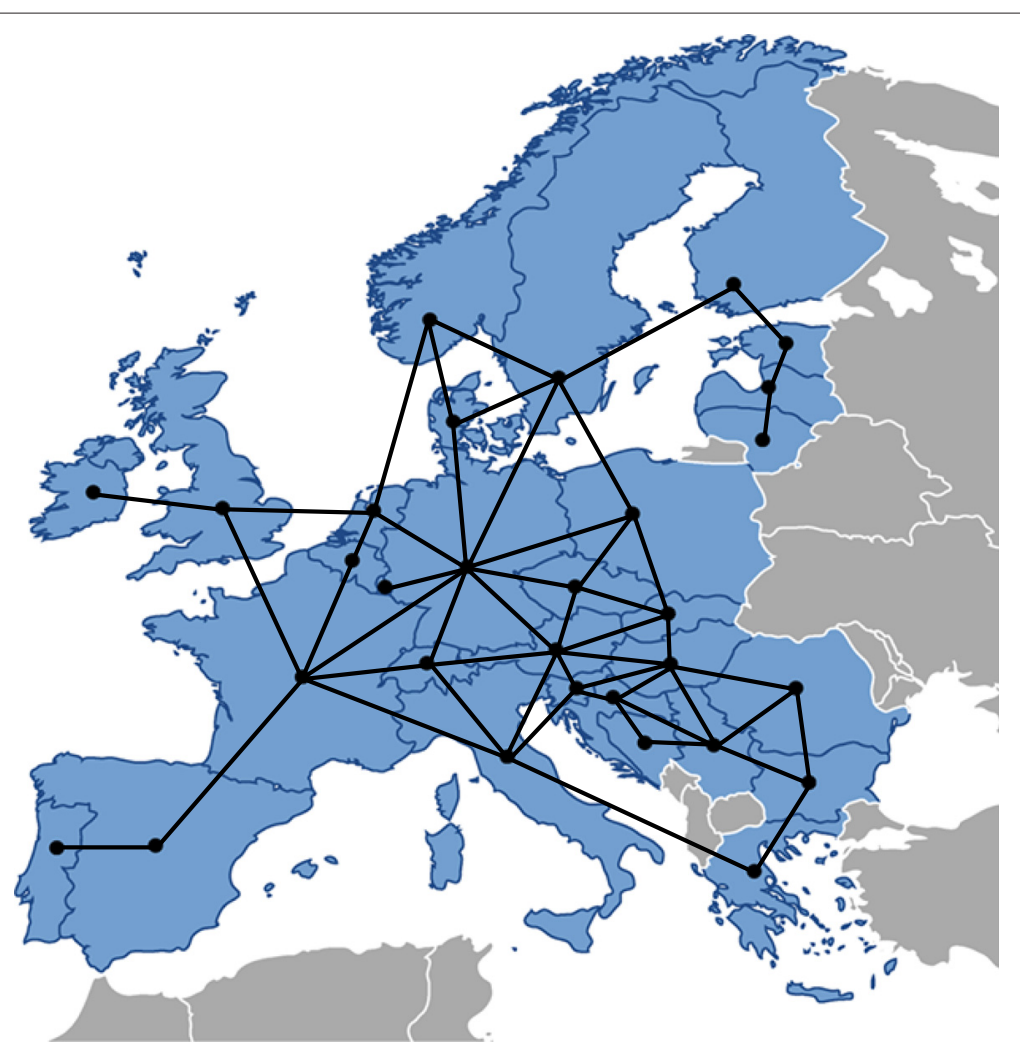

Fig. 2 Pan-European transmission network. A simplified pan-European transmission network, where countries are treated as ideal nodes and are linked by interconnectors

defines the mixing parameter between wind and solar power generation. Furthermore, we assume that intracountry transmission capacities are sufficient to allow for aggregation of load and generation on country level.

Realistic time series for the wind and solar PV power generation, including consistent spatio-temporal correlations, have been obtained from a recently developed renewable energy atlas [22]. The atlas is based on weather data from high-resolution forecast models. The data extends over several years with hourly time resolution and has a spatial grid cell resolution of $0.3125^{\circ} \times 0.3125^{\circ}$ (approximately $40 \times 40 \mathrm{~km}^{2}$ at European latitudes) covering all of Europe. The conversion from weather data to potential wind and solar PV power generation is done on a grid cell level and then aggregated to a country level. The load time series $L_{n}(t)$ have been taken from historical records with hourly resolution. For more details on the wind, solar, and load data, see $[11,12]$.

\section{Backup energy and power}

Although equal on average, the renewable power generation of a fully renewable country will be larger than the load for roughly half of the time, and smaller for the other half. The country then might want to export $\left(T_{n}>0\right)$ or import $\left(T_{n}<0\right)$ power. This defines the residual mismatch $\Delta_{n}(t)$ normalized to the mean load $\left\langle L_{n}\right\rangle$ :

$$
\Delta_{n}(t)=G_{n}^{\mathrm{RES}}(t)-L_{n}(t)-T_{n}(t) .
$$

If $\Delta_{n}(t)$ is negative, the country needs to balance $B_{n}(t)=-\min \left(\Delta_{n}(t), 0\right)$ with own backup power plants, and if positive, the country needs to curtail $C_{n}(t)=$ $\max \left(\Delta_{n}(t), 0\right)$.

As mentioned above, dispatchable renewable generation, such as hydro power or biomass, is considered to be a part of backup power. We do not take into account any inertia of the backup system due to finite reaction times. Instead, we consider it to be ideally flexible. For a more detailed treatment of the backup system, distinguishing between a range of flexibility classes of a weather-driven backup power system, see [25, 26].

Due to fluctuations in the renewable power generation, the load and the export power, the backup power $B_{n}(t)$ changes from $1 \mathrm{~h}$ to the next. When multiplied with the number of hours $N_{\text {hours }}=8760$ per year, the average backup power is equal to the annual backup energy $E_{n}^{B}=$ $\left\langle B_{n}\right\rangle N_{\text {hours. }}$. We take the $99 \%$ quantile $\int_{0}^{\mathcal{K}^{B}} p(B) d B=0.99$ of the backup power distribution as a measure for the 
backup capacity $\mathcal{K}_{n}^{B}$, as rare, extreme values define the $100 \%$ quantile.

Splitting the residual mismatch from Equation (3) into backup and curtailment leads to the energy conservation law of power supply matching power demand

$$
G_{n}^{\mathrm{RES}}(t)+B_{n}(t)=L_{n}(t)+T_{n}(t)+C_{n}(t),
$$

which holds locally at every country. Based on the assumption of lossless transmission, a second conservation law holds globally for the networked countries:

$$
\sum_{n} T_{n}(t)=0 .
$$

\section{Power transmission}

In the DC approximation [27] for high-voltage systems, the AC power flows along the interconnectors between the countries are directly determined from the net exports:

$$
\begin{aligned}
T_{n} & =\sum_{m=1}^{N} \mathcal{B}_{n m} \delta_{m}, \\
f_{m \rightarrow n} & =b_{n m}\left(\delta_{n}-\delta_{m}\right) .
\end{aligned}
$$

Inversion of the first equation gives the voltage phase angles $\delta_{n}$ at all nodes. Their differences determine the power flows $f_{m \rightarrow n}$ along the links $m \rightarrow n$ between neighboring nodes (negative flows are used to denote flow in the direction $n \rightarrow m$ ). To reduce the complexity of a full solution to the $\mathrm{AC}$ problem, the $\mathrm{DC}$ approximation assumes zero link resistances and no reactance dependence on link lengths. The elements of the susceptance matrix $\mathcal{B}_{n m}=\left(\sum_{k} b_{n k}\right) \delta_{n m}-b_{n m}$ are therefore initially assumed to be unitary and equal to the Laplacian of the network graph; see [14] for more details.

To examine the validity of the distance-independent line susceptances, we calculated test cases. There, we assume non-unitary reactance values, using instead a typical value of $0.28 \Omega / \mathrm{km}$ [28]. As a metric for the line distance, we use the distance between capitals of the countries in question. This leads to $b_{n m}=0.28 \cdot d_{n m}$ where $d_{n m}$ is the line distance. Given that 0.28 is a linear factor, the actual number is irrelevant, and the only effect it brings is a preference for shorter paths over longer ones. In terms of total transmission capacities, this leads to a slight increase by about $9 \%$.

Since the exports $T_{n}(t)$ are functions of time, the power flows $f_{l}(t)$ will also fluctuate with time. The low and high quantiles $q=\int_{-\infty}^{F(q)} p(f) d f$ of the unconstrained flow distributions $p(f)$ represent a measure for the required interconnector strengths, which we set to $\mathcal{K}_{l}^{T}=\max \left(-F_{l}(0.01), F_{l}(0.99)\right)$. The total interconnector capacity $\mathcal{K}^{T}=\sum_{l} \mathcal{K}_{l}^{T}$ of the pan-European transmission network is given by the sum over all links.

\section{Export schemes}

Next, four different export schemes are outlined. They include zero export, two localized trade schemes of only renewable excess power, and synchronized backup operation with additional export of backup power.

\section{Zero export}

The zero-export scheme is expressed by

$$
T_{n}=0,
$$

and is illustrated in Fig. 1a. Each country then has to fully balance or curtail the mismatch between its renewable power generation and its load.

\section{Localized export I}

Rather than full curtailment, countries with surplus renewable power generation may want to export as much of it as possible to other countries with a current deficit:

$$
\begin{aligned}
& \text { exporters : } 0<T_{n}(t) \leq \max \left(G_{n}^{\mathrm{RES}}(t)-L_{n}(t), 0\right), \\
& \text { importers : } 0>T_{n}(t) \geq \min \left(G_{n}^{\mathrm{RES}}(t)-L_{n}(t), 0\right), \\
& \text { objective 1: } \min \left(\sum_{n} B_{n}(t)\right), \\
& \text { objective 2: } \min \left(\sum_{l} f_{l}^{2}(t)\right) .
\end{aligned}
$$

Two steps need to be taken to assign exporters and importers. The first objective is to use as much excess renewable power as possible to reduce the overall backup energy. In case that the overall export strengths are larger than the overall import needs, this objective makes sure that all importers will be fully served, but there is still the freedom to select different subsets of exporters. This freedom is fixed by the second objective, where the transmission system operator requires the power flows on the network to be as localized as possible. Those nodes which are at the end left unable to export everything have to locally curtail.

In case of larger overall import needs, all export opportunities will be used by the first objective, and the second objective fixes the subset of importers. Those nodes which are not able to import enough then have to locally balance for themselves. Compare again with Fig. 1b. Note also that from one time instance to the next, the role of exporters and importers can be reversed.

This export scheme has been employed in two previous publications [14, 20]. Given that generation from renewables has a lower marginal cost, it is not unrealistic that importers would be willing to import excess renewable power generation from other nodes. Excessive transmission capacities would increase the total system cost. A localization of exports-as forced by the square minimization of total flows-does result in smaller total transmission capacities, as shown in the results below. 


\section{Localized export II}

The third export scheme uses the same constraints on exporters and importers as in (8), but replaces the first objective by

$$
\text { objective 1: } \min \left(\max _{n} \frac{B_{n}(t)}{\left\langle L_{n}\right\rangle}\right) .
$$

This gives priority to importers with the largest relative deficit power, allowing reductions to the backup capacities. The second step is, again, identical to the one in (8). Compare with Fig. 1c.

\section{Synchronized export}

A very different export scheme forces all countries into a completely synchronized residual mismatch (3):

$$
\Delta_{n}(t)=\beta(t)\left\langle L_{n}\right\rangle,
$$

where due to (3) and (5)

$$
\beta(t)=\frac{\sum_{n}\left(G_{n}^{\mathrm{RES}}(t)-L_{n}(t)\right)}{\sum_{n}\left\langle L_{n}\right\rangle} .
$$

After exports and imports, every node is balancing $(\beta<0)$ or curtailing $(\beta>0)$ the same amount relative to its own average load as all the other nodes. This requires cooperation. For example in case of $\beta<0$, nodes with excess renewable power generation not only export their excess to other countries but also balance power from their own backup capacities; see again Fig. 1d.

In the zero export case, the backup capacities are determined by the countries' weather patterns and demand. For the localized export schemes, the position of the country in the network also affects its backup capacity. An immediate result of the synchronized export scheme is a uniform distribution of backup capacities, relative to the countries' mean load.

All export schemes can be stated as a quadratic optimization problem and were computed numerically using the python interface to the commercial solver Gurobi [29].

\section{Results and discussion}

\section{Residual mismatch distributions}

The distribution $p\left(\Delta_{n}\right)$ of the residual mismatch (3) depends strongly on the mixing parameter $\alpha_{n}$ between wind and solar power generation, as previously discussed in [14]. Lowest backup energy needs can be obtained with $\alpha_{n} \approx 0.7$ in single countries. This value of the mixing parameter is therefore used in the subsequent analysis. Although zero on average, the distributions are very broad, cf. Fig. 3, showing the distribution of non-zero mismatches for the different export schemes.

The negative part of the mismatch distribution is of particular importance. Its average determines the average backup power, which is proportional to the annual backup energy. Its tail characterizes the most extreme backup

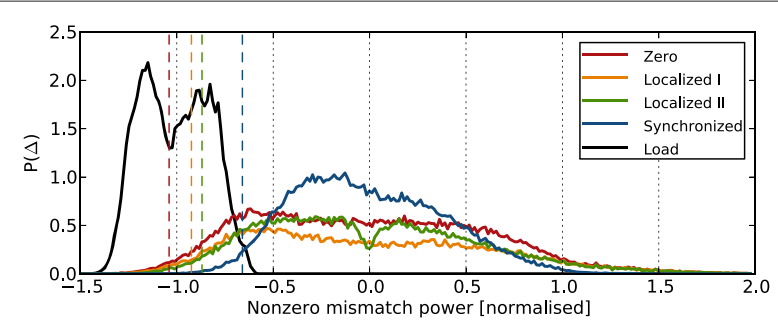

Fig. 3 Residual mismatch distributions. Distributions of residual mismatches (3) for Germany with $\alpha_{\mathrm{GER}}=0.7$ resulting from the zero (red), the two localized (yellow, green) and the synchronized (blue) export schemes. For comparison, the negative load distribution (black) is also shown. Mismatches and load have been normalized to the average load $\left\langle L_{G E R}\right\rangle$. The residual mismatch distributions resulting from the two localized export schemes are shown without their singular peak at $\Delta_{\mathrm{GER}}=0$. Dashed vertical lines indicate the $99 \%$ quantile of the negative mismatch

power needs, which determine the required backup capacities.

Figure 3 shows the dependence of the residual mismatch distribution on the three non-zero export schemes (8)-(10), where each country is allowed to import and export from all 30 European countries. For comparison, the load and the zero export schemes are shown as well. Contrary to the synchronized export scheme, the two localized schemes produce a lot of $\Delta_{n}=0$ events. The resulting singular peak has been removed from the plotted distributions to clarify the illustration. It can still be observed indirectly as the integrated area below those two normalized distributions is smaller than for the other two distributions resulting from the zero and the synchronized export scheme.

The total backup energy (or equivalently, the average backup power) is shifted to smaller values by transmission. In fact, as transmission line capacities are not constrained, all non-zero export schemes lead to the same total backup energy, equal to the backup needs of a European aggregation. This is because in the event of a Europe-wide deficit, all local excesses are exported to other countries in deficit and none are curtailed. Conversely, when there is Europe-wide excess, all local deficits are served before curtailment is activated. In the two localized schemes, this is achieved by the first objective of minimal backup energy needs. In the synchronized export scheme, it is a consequence of the Europe-wide aggregation of the mismatch, which is then distributed back to the single nodes as backup or curtailment, cf. (10). When compared to the zero export scheme, the two localized export schemes are able to lower the frequency of negative residual mismatches, causing a decrease of the average backup power. The residual mismatch distribution resulting from the synchronized export scheme changes in a different way, also leading to a reduction of the average backup power. 
Contrary to the two localized export schemes, the synchronized export scheme also lowers the negative tail of the residual mismatch distribution and therefore significantly reduces the required backup capacity. As a proxy to the backup capacity, the $99 \%$ quantile of the negative mismatches is indicated as dashed vertical lines in Fig. 3.

\section{Backup energy and capacity}

The two localized and the synchronized export schemes (8)-(10) have a different impact on the average backup power for the different countries. See the top panel of Fig. 4, which shows the average imports $\left\langle\max \left(-T_{n}, 0\right)\right\rangle$ for all countries. For example, Germany, the first localized export scheme leads to a slightly larger reduction of the average backup power when compared to the second localized scheme. This is because the first scheme allows Germany to import more on average than the second scheme. The synchronized export scheme leads to a significantly larger average import. However, this does not lead to a further reduction of the average backup power because each country's exports increase in parallel.

For some countries other than Germany, the two localized export schemes (8)-(9) lead to a smaller average backup power than from the synchronized export scheme (10). This is explained by the observation that the sum $\sum_{n}\left\langle B_{n}\right\rangle$ is identical for the three export schemes as discussed above. Consult also again Fig. 1b-d.

The lower panel of Fig. 4 shows the average net exports $\left\langle T_{n}\right\rangle=-\left\langle\Delta_{n}\right\rangle$ for the different countries. The average net export is the difference between average export and average import. Except for a minus sign, it is equal to the mean of the residual mismatch (3). For the synchronized export scheme (10), the net exports are zero for all countries. However, this is not the case for the two localized export schemes (8) and (9). For big countries like Germany, France, and Great Britain with $\alpha_{n}=0.7$, it turns out to be of the order $\left\langle T_{n}\right\rangle /\left\langle L_{n}\right\rangle \approx-0.004$. Those countries become net importers. The biggest relative net importers are the two Nordic countries Norway and Sweden with $\left\langle T_{n}\right\rangle /\left\langle L_{n}\right\rangle \approx-0.023$. Spain and Italy are net exporters of the order $\left\langle T_{n}\right\rangle /\left\langle L_{n}\right\rangle \approx 0.014$. With $\left\langle T_{n}\right\rangle /\left\langle L_{n}\right\rangle \approx 0.023$, the strongest relative net exporters are the smaller countries in southeast Europe. Of course due to (5), the overall country sum over all net exports and net imports is again $\sum_{n}\left\langle T_{n}\right\rangle=0$.

The backup capacity $\mathcal{K}_{n}^{B}$ also shows a strong dependence on the mixing parameter. See Fig. 5. For all four export schemes (7)-(10), it takes a minimum at $\alpha_{n}=0.85$. Compared to the value $1.32\left\langle L_{n}\right\rangle$ of the $99 \%$ quantile of the German load, the backup capacity $\mathcal{K}_{n}^{B}=1.04\left\langle L_{n}\right\rangle$ at $\alpha_{n}=0.85$ resulting from the zero export rule is not much smaller. Without transmission, a $100 \%$ renewable country needs an enormous amount of backup capacity. The two localized and the synchronized export schemes have a bigger impact on the reduction of the backup capacities. For Germany with $\alpha=0.85$, the first localized export scheme leads to $\mathcal{K}_{n}^{B}=0.89\left\langle L_{n}\right\rangle$, the second localized scheme to $\mathcal{K}_{n}^{B}=0.82\left\langle L_{n}\right\rangle$, and the synchronized scheme

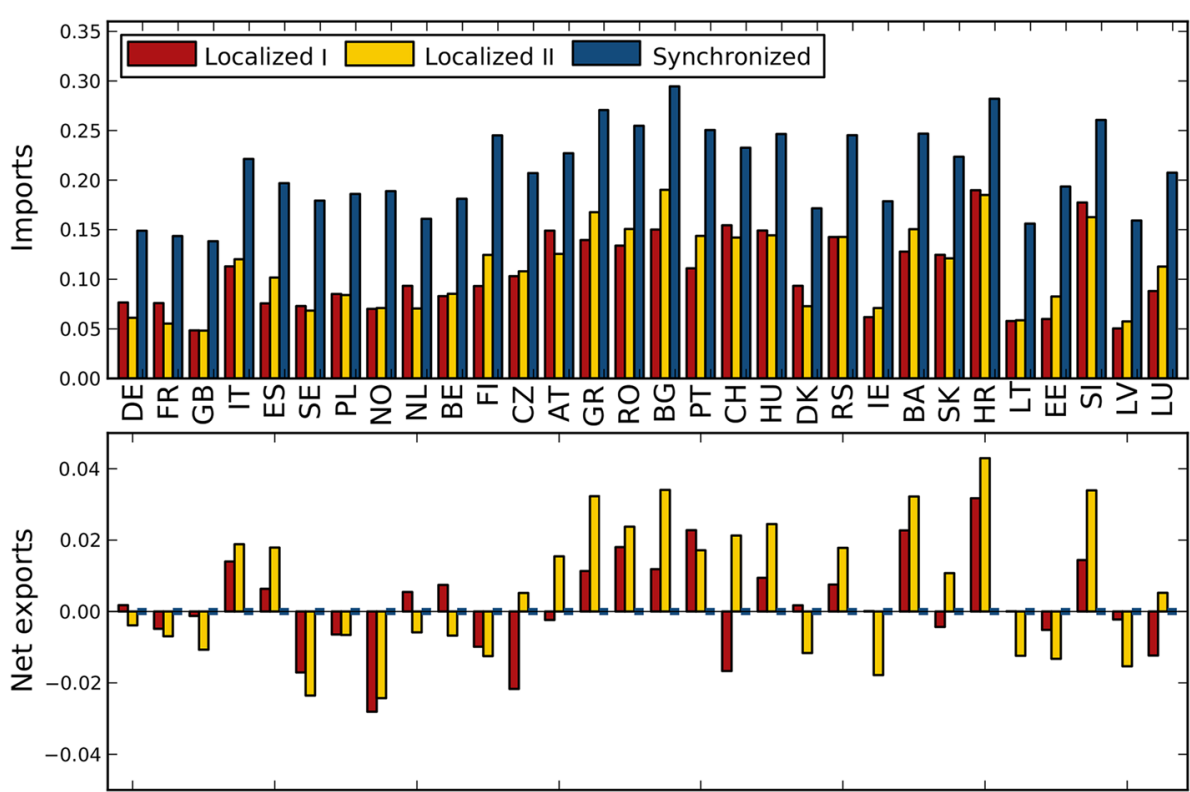

Fig. 4 Average imports and net exports. (Top) average imports $\left\langle\max \left(-T_{n}, 0\right)\right\rangle$ and (bottom) average net exports $\left\langle T_{n}\right\rangle$ for all countries following from the two localized and the synchronized export schemes (8)-(10). The renewable mixing parameter $\alpha_{n}=0.7$ has been used, and the imports and net exports have been normalized to the average loads $\left\langle L_{n}\right\rangle$ 


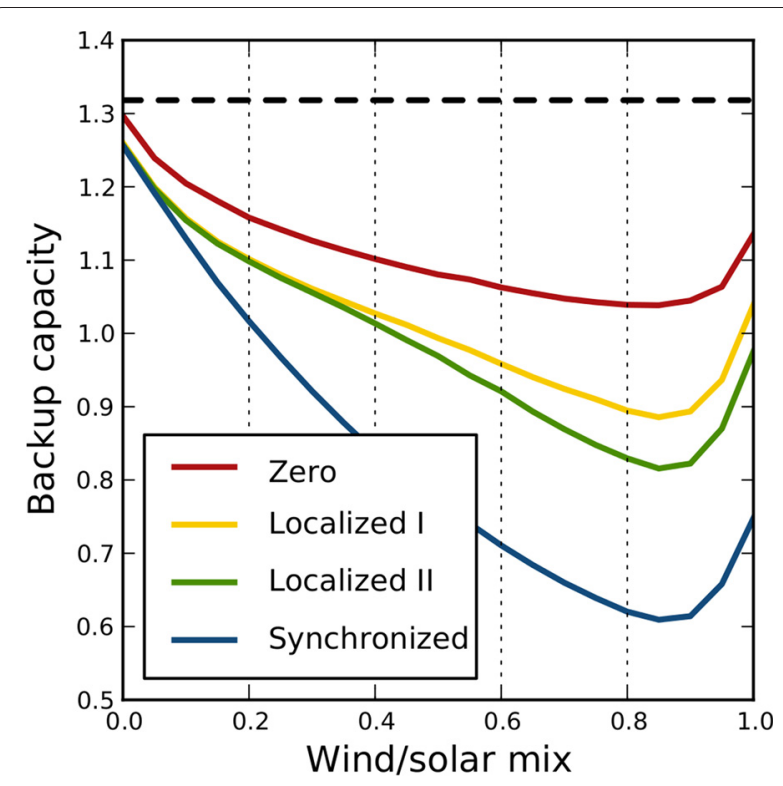

Fig. 5 Backup capacity. Backup capacity as a function of the renewable mixing parameter $\alpha_{\mathrm{GER}}$ for Germany resulting from four different export schemes. The backup capacity has been normalized to the average load $\left\langle L_{G E R}\right\rangle$. For reference, the $99 \%$ quantile of the German load distribution is shown as the black dashed line

to $\mathcal{K}_{n}^{B}=0.61\left\langle L_{n}\right\rangle$. For the other countries, the relative backup capacities resulting from the two localized export schemes are slightly different than the German values (see Fig. 6). The synchronized export scheme leads to identical relative backup capacities for all countries. This is no surprise since the synchronized export scheme (10) with (11) enforces a synchronized balancing between all countries.

Since Equation (11) describes the all-European mismatch, we arrive at a strong conclusion: the synchronized export scheme leads to the largest possible reduction in overall backup capacities. More precisely, synchronized export takes advantage of the strongest possible mismatch smoothing by aggregation across Europe. In the absence of single countries with exceptionally stable resources that would make such sharing unattractive (see Fig. 6) and without countries taking advantage of each other (in game-theoretic terms, as long as free-riding and altruistic behavior are excluded), there is no scheme in which the mismatch quantiles of single countries can be decreased more. In particular, no other export scheme is able to reduce the backup capacities (measured as quantiles of the mismatch) further. For mixes between $0.70 \leq \alpha_{n} \leq 0.95$, the synchronized export scheme leads to backup capacities which are only half of the high load quantiles, the latter representing more or less the backup capacities of our current electricity system.

\section{Transmission capacities}

Reference [14] has shown that the unconstrained total interconnector capacities become relatively large. The same European country network as in Fig. 2 has been used with a mixing parameter around $\alpha_{n} \approx 0.71$, and only the first localized export scheme has been discussed. For unitary impedance across the whole network, the total interconnector capacities turned out to be $\mathcal{K}^{T}=395$ GW, which is about a factor 5.4 larger than the present European layout; see Table two in [14].

Figure 7 extends the previous findings to the full range $0 \leq \alpha_{n} \leq 1$ of the mixing parameter, and to the second localized and the synchronized export schemes. The two localized export schemes produce very similar total interconnector capacities. The minimum capacities are reached at $\alpha_{n}=0.35$. For the solar only $\alpha_{n}=0$, the transmission capacities are larger because of the strong East-West power flows in the mornings and evenings, and because of the strong seasonal South-North flows. For the other extreme, the wind-only $\alpha_{n}=1$, the transmission capacities become even larger. This is caused by strong power flows between decorrelated regions separated by distances larger than the observed correlation length of about $500 \mathrm{~km}$ for the wind power generation $[15,18]$.

The synchronized export scheme leads to a minimum at a slightly larger $\alpha_{n}=0.42$. Over the whole range $0 \leq$

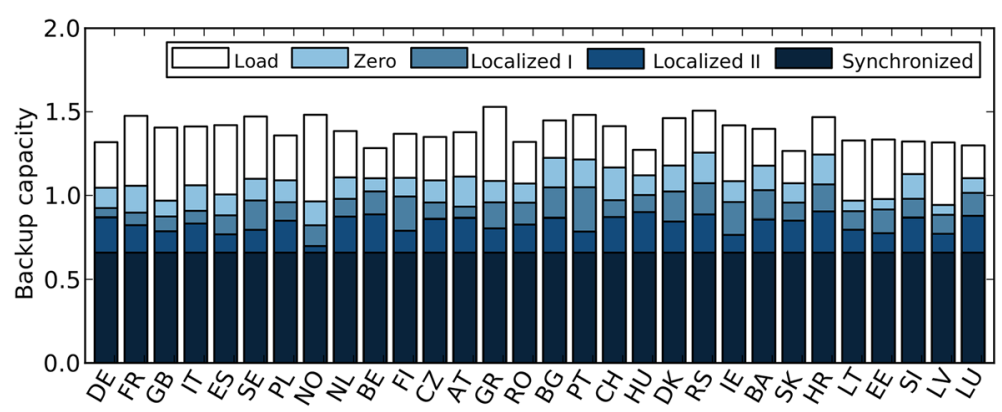

Fig. 6 European backup capacities. Backup capacities for all European countries resulting from four different export rules. The renewable mixing parameter $\alpha_{n}=0.7$ has been used, and the backup capacities have been normalized to the average loads $\left\langle L_{n}\right\rangle$. For reference, the $99 \%$ quantile of the load distributions is also shown 


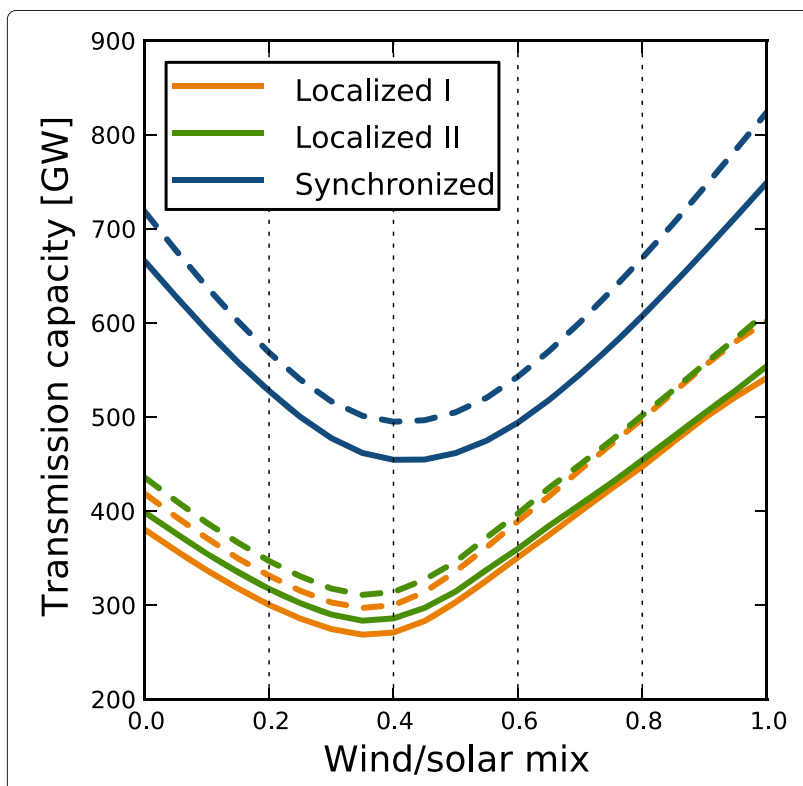

Fig. 7 Transmission capacity. Overall interconnector capacities $\boldsymbol{\mathcal { K }}^{\top}$ of the unconstrained European transmission grid as a function of the renewable mixing parameter $\alpha_{n}$. Dashed lines indicate $\mathcal{K}^{T}$ for non-unitary, line-dependent impedances. The scale of $\mathcal{K}^{T}$ is set by the average European load $\sum_{n}\left\langle L_{n}\right\rangle=345.5 \mathrm{GW}$ from the year 2007

$\alpha_{n} \leq 1$, the total interconnector capacities required from the synchronized scheme are a factor of 1.35-1.70 larger than for the two localized schemes. For $\alpha_{n}=0.7$, the synchronized scheme produces $\mathcal{K}^{T}=550 \mathrm{GW}$, whereas it is only $\mathcal{K}^{T}=400 \mathrm{GW}$ for the localized schemes. This difference is easily explained by the extra exports of backup power caused by the synchronized scheme (see again Fig. 4), which cause more power flows and result in higher required transmission capacities (see again Figs. $1 \mathrm{~b}-\mathrm{d}$ ).

Reproducing the results considering non-unitary, length-dependent impedances for all lines brings only small changes to the results. Quantitatively, total backup energy and capacity are unchanged, as the objective that minimizes them has priority over the actual flow minimization. Since longer paths are more penalized in this calculation, the total amount of capacity needed is higher, but only by around $9 \%$, regardless of the export scheme used. The dashed lines in Fig. 7 show the transmission capacities resulting from calculating flows with impedance.

\section{Conclusions}

Fully renewable networked power systems have been discussed, for which the average renewable power generation is equal to the average load. Such systems still require backup generation units to cover the occurring temporal deficits of fluctuating renewable power generation to match the load. The amount of backup capacity depends on the export schemes applied to the transmission network. Compared to no exports at all, the export of local renewable excess power, which we have dubbed as localized, leads to some reduction of the overall backup capacities. Significantly more reduction is achieved when also local backup power is allowed to be exported. For a fully renewable pan-European transmission grid, such a synchronized export scheme leads to backup capacities which are only half of the maximum loads.

In its present form, the synchronized export scheme is based on a completely synchronized residual mismatch across the network. No other export scheme is able to reduce the backup capacities further. However, it requires larger transmission capacities when compared to the localized export schemes. From an economic perspective, this is not necessarily a drawback, since costs for backup capacities are usually one order of magnitude bigger than transmission costs [3]. It will be interesting to explore other, partly synchronized export schemes, including market-like schemes, which might be able to reduce also the transmission capacities. Such an exploration could be based on the minimization of an overall cost objective, which includes not only backup and transmission capacities but also renewable power generation capacities. These findings will have an impact on the future strategies of policymakers and energy-related companies.

\section{Competing interests}

The authors declare that they have no competing interests.

\section{Authors' contributions}

The article was jointly prepared by all authors. All authors read and approved the final manuscript.

\section{Acknowledgements}

SB gratefully acknowledges financial support from O. and H. Stöcker as well as M. and H. Puschmann.

\section{Author details}

${ }^{1}$ Department of Mathematics, Aarhus University, Ny Munkegade 118, 8000 Aarhus C, Denmark. ${ }^{2}$ Department of Physics and Astronomy, Aarhus University, Ny Munkegade 120, 8000 Aarhus C, Denmark. ${ }^{3}$ Frankfurt Institute for Advanced Studies, Frankfurt University, Ruth-Moufang-Str.1, 60438 Frankfurt am Main, Germany. ${ }^{4}$ Department of Engineering, Aarhus University, Inge Lehmanns Gade 10, 8000 Aarhus C, Denmark.

Received: 18 February 2014 Accepted: 29 May 2015

Published online: 07 July 2015

\section{References}

1. Jacobson MZ, Delucchi MA (2009) A path to sustainable energy by 2030. Sci Am 301:58-65

2. Jacobson MZ, Delucchi MA (2011) Providing all global energy with wind, water, and solar power - Part I: technologies, energy resources, quantities and areas of infrastructure, and materials. Energy Policy 39:1154-1169

3. Delucchi MA, Jacobson MZ (2011) Providing all global energy with wind, water, and solar power - Part II: reliability, system and transmission costs, and policies. Energy Policy 39:1170-1190

4. Popp M (2010) Speicherbedarf Bei Einer Stromversorgung Mit Erneuerbaren Energien. Springer, Heidelberg 
5. Lund H (2010) Renewable Energy Systems - The Choice and Modeling of $100 \%$ Renewable Solutions. Elsevier, Amsterdam

6. Connolly D, Lund H, Mathiesen B, Leahy M (2011) The first step towards a $100 \%$ renewable energy system for Ireland. Appl Energy 88:502-507

7. Mason IG, Page SC, Williamson AG (2010) A $100 \%$ renewable electricity system for New Zealand utilising hydro, wind, geothermal and biomass resources. Energy Policy 38:3973-3984

8. Mason IG, Page SC, Williamson AG (2013) Security of supply, energy spillage control and peaking options within a $100 \%$ renewable electricity system for New Zealand. Energy Policy 60:324-333

9. Elliston B, Diesendorf M, MacGill I (2012) Simulations of scenarios with $100 \%$ renewable electricity in the Australian National Electricity Market. Energy Policy 45:606-613

10. Elliston B, Diesendorf M, MacGill I (2013) Least cost $100 \%$ renewable electricity scenarios in the Australian National Electricity Market. Energy Policy 59:270-282

11. Heide D, Von Bremen L, Greiner M, Hoffmann C, Speckmann M, Bofinger S (2010) Seasonal optimal mix of wind and solar power in a future, highly renewable Europe. Renew Energy 35:2483-2489

12. Heide D, Greiner M, Von Bremen L, Hoffmann C (2011) Reduced storage and balancing needs in a fully renewable European power system with excess wind and solar power generation. Renew Energy 36:2515-2523

13. Rasmussen MG, Andresen GB, Greiner M (2012) Storage and balancing synergies in a fully or highly renewable pan-European power system. Energy Policy 51:642-651

14. Rodríguez RA, Becker S, Andresen GB, Heide D, Greiner M (2014) Transmission needs across a fully renewable European power system. Renew Energy 63:467-476

15. Widen J (2011) Correlations between large-scale solar and wind power in a future scenario for Sweden. IEEE Trans Sustainable Energy 2:177-184

16. Schaber K, Steinke F, Hamacher T (2012) Transmission grid extensions for the integration of variable renewable energies in Europe: Who benefits where? Energy Policy 43:123-135

17. Steinke F, Wolfrum P, Hoffmann C (2013) Grid vs. storage in a $100 \%$ renewable Europe. Renew Energy 50:826-832

18. Kempton W, Pimenta FM, Veron DE, Colle BA (2010) Electric power from offshore wind via synoptic-scale interconnection. Proc Natl Acad Sci 107:7240-7245

19. Budischak C, Sewell D, Thomson H, Mach L, Veron DE, Kempton W (2013) Cost-minimized combinations of wind power, solar power and electrochemical storage, powering the grid up to $99.9 \%$ of the time. J Power Sources 225:60-74

20. Becker S, Rodriguez RA, Andresen GB, Schramm S, Greiner M (2014) Transmission grid extensions during the build-up of a fully renewable pan-European electricity supply. Energy 64:404-418

21. Andresen GB, Rasmussen MG, Rodriguez RA, Becker S, Greiner M (2012) Fundamental properties of and transition to a fully renewable pan-European power system. EPJ Web of Conferences 33

22. Andresen G, Søndergaard A, Rodriguez RA, Becker S, Greiner M (2013) Weather-driven modeling of future renewable power systems. In Proceedings of the 8th SDEWES Conference; Dubrovnik, Croatia

23. Schäfer M, Scholz J, Greiner M (2006) Proactive robustness control of heterogeneously loaded networks. Phys Rev Lett 96:108701

24. Heide D, Schäfer M, Greiner M (2008) Robustness of networks against fluctuation-induced cascading failures. Phys Rev E (Stat Nonlinear, Soft Matter Phys) 77:056103

25. Andresen G, Rasmussen MG, Rodriquez RA, Becker S, Greiner M (2012) Fundamental properties of and transition to a fully renewable pan-European power system. In: 2nd European Energy Conference; Maastricht, The Netherlands. Vol. 33. p 04001

26. Andresen G, Heide D, Rasmussen M, Greiner M (2011) Transition to a fully renewable power system in Europe. In: Proceedings of the 10th International Workshop on Large-Scale Integration of Wind Power Into Power Systems as Well as on Transmission Networks for Offshore Wind Power Plants. Aalborg University, Aalborg, Denmark. pp 322-325
27. Wood AJ, Wollenberg BF (1996) Power Generation, Operation and Control. John Wiley \& Sons, New York

28. Zhou Q, Bialek JW (2005) Approximate model of european interconnected system as a benchmark system to study effects of cross-border trades. Power Syst IEEE Trans 20(2):782-788

29. Gurobi Optimization I (2014) Gurobi Optimizer Reference Manual. http://www.gurobi.com

\section{Submit your manuscript to a SpringerOpen ${ }^{\circ}$ journal and benefit from:}

- Convenient online submission

- Rigorous peer review

- Immediate publication on acceptance

- Open access: articles freely available online

- High visibility within the field

- Retaining the copyright to your article

Submit your next manuscript at $\boldsymbol{\nabla}$ springeropen.com 